\title{
Electron beam-induced nanopatterning of multilayer graphene and amorphous carbon films with metal layers
}

Julio A. Rodríguez-Manzo, and Florian Banhart

Citation: Appl. Phys. Lett. 98, 183105 (2011); doi: 10.1063/1.3587634

View online: https://doi.org/10.1063/1.3587634

View Table of Contents: http://aip.scitation.org/toc/apl/98/18

Published by the American Institute of Physics

Articles you may be interested in

Electron beam nanosculpting of suspended graphene sheets

Applied Physics Letters 93, 113107 (2008); 10.1063/1.2980518 


\title{
Electron beam-induced nanopatterning of multilayer graphene and amorphous carbon films with metal layers
}

\author{
Julio A. Rodríguez-Manzo and Florian Banhart ${ }^{\mathrm{a}}$ \\ Institut de Physique et Chimie des Matériaux, UMR 7504 CNRS, Université de Strasbourg, \\ 23 rue du Loess, 67034 Strasbourg, France
}

(Received 24 February 2011; accepted 15 April 2011; published online 4 May 2011)

\begin{abstract}
Thin Co and Ni lamellae grow under electron irradiation of metal crystals supported on multilayer graphene or amorphous carbon films. The lateral growth of a lamella from a source crystal is achieved by directing an electron beam to the periphery of the metal crystal and moving the beam over the surrounding carbon. Patterns of linear, branched, or ringlike metal lamellae can be created. The patterning is carried out in situ in a transmission electron microscope, allowing simultaneous structuring and imaging. The process is driven by the metal-carbon interaction at a beam-activated carbon surface. (c) 2011 American Institute of Physics. [doi:10.1063/1.3587634]
\end{abstract}

Focused electron beams can be used as tools to deposit or etch materials with a spatial precision at the nanoscale. ${ }^{1-3}$ Transmission electron microscopes (TEMs), usually working in the 100-300 keV energy range, can be conveniently used and allow combined structuring, imaging, and analysis. ${ }^{4}$ The techniques of electron beam-induced nanostructuring ${ }^{1-3}$ have been demonstrated, e.g., for patterning metal structures by ablation $^{5}$ or to deposit metals by electron beam-induced deposition. ${ }^{6}$ The direct beam-metal interaction has been used, e.g., for drilling nanoholes into a metal layer, ${ }^{7,8}$ creating metal nanobridges, ${ }^{9}$ or sculpting nanoelectrodes. ${ }^{10}$ Here we report a technique of growing thin Co or Ni lamellae by an electron irradiation-induced transport of metal atoms, starting from metal crystals on amorphous or graphenelike carbon substrates.

Free-standing amorphous carbon films with a thickness of approximately $20 \mathrm{~nm}$, spanning over the holes of standard TEM Cu grids (Agar Scientific Ltd.), were used as substrates for the deposition of thin $\mathrm{Co}, \mathrm{Ni}, \mathrm{Cu}$, or Pt layers. ${ }^{11}$ The deposition was carried out in a cathodic sputtering chamber with an argon atmosphere $(0.1 \mathrm{~Pa})$ at room temperature, resulting in coherent polycrystalline layers of $10-20 \mathrm{~nm}$ in thickness on the carbon films. The samples were mounted in the heating stage of a TEM (Jeol 2100F, operated at $200 \mathrm{kV}$ and equipped with an aberration-corrected condenser). The metal-carbon bilayer films were first heated up to $500{ }^{\circ} \mathrm{C}$ in the TEM, causing coalescence of metal crystallites and formation of thicker isolated metal islands by Ostwald ripening ${ }^{12}$ and leaving uncovered regions on the carbon film between the metal crystals. Carrying out the annealing at temperatures above $600{ }^{\circ} \mathrm{C}$ caused the transformation of the amorphous carbon film under the metal crystals to multilayer graphene. ${ }^{13}$ After the annealing, the electron beam was converged to a spot of approximately $3 \mathrm{~nm}$ in diameter, carrying a current density of $10^{4} \mathrm{~A} \mathrm{~cm}^{-2}$, and directed onto the metal particles at temperatures of $450-600{ }^{\circ} \mathrm{C}$. While the metal crystals remained almost unchanged when the beam spot was on the center of the particles, irradiation of the edges caused a growth of the metal in the shape of a thin lamella in the direction of the beam spot.

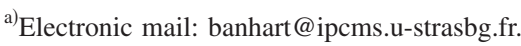

The growth of a metal lamella was observed to start from $\mathrm{Co}$ and $\mathrm{Ni}$ islands and proceeding over the carbon film but not beyond the area under the electron beam. When the beam spot was slowly moved away from the metal island, the growing metal layer followed the beam so that a long and thin metal lamella was left on the trace, as shown in Figs. 1(a)-1(c). Lateral growth rates of tens of nanometer per minute were achieved. By moving the beam in predefined

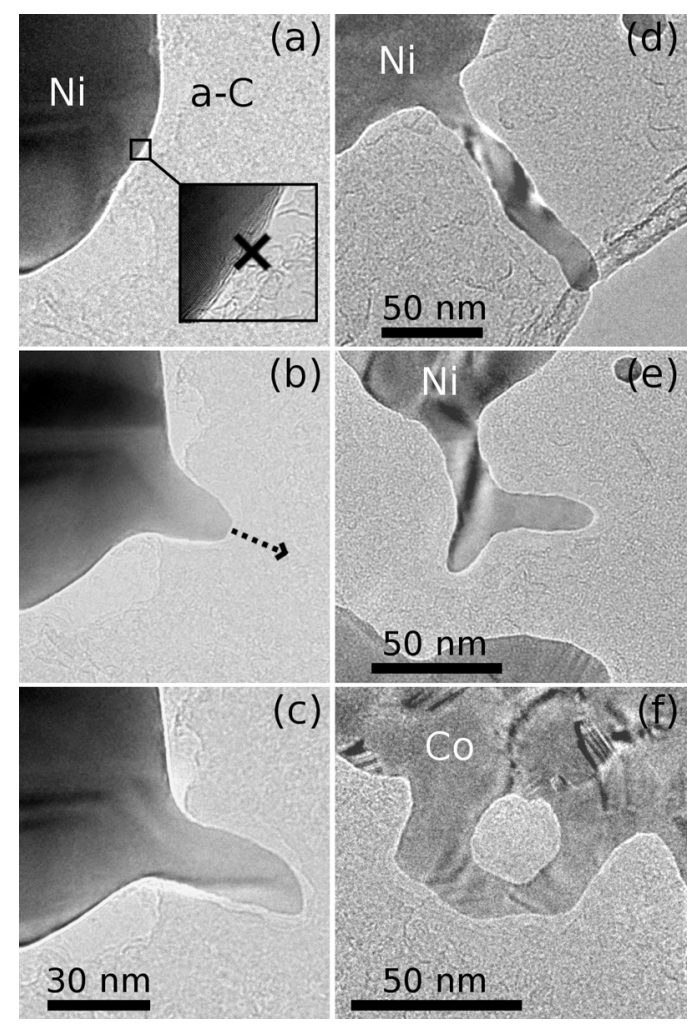

FIG. 1. [(a)-(c)] Formation of a Ni lamella by controlled electron irradiation of the edge of a Ni crystal on an amorphous carbon film at $560{ }^{\circ} \mathrm{C}$. (a) The beam spot was moved from its starting position (marked with a cross in the inset) away from the metal particle. A thin Ni lamella followed the beam (b) until, after $10 \mathrm{~min}$, (c) a metal lamella (20 nm wide and $45 \mathrm{~nm}$ long) was obtained. [(d)-(f)] Examples of metal patterns formed by moving the electron beam spot in predefined directions. (d) $120 \mathrm{~nm}$ long Ni lamella formed at $550{ }^{\circ} \mathrm{C}$. (e) Three-terminal structure formed with $\mathrm{Ni}$ at $550{ }^{\circ} \mathrm{C}$. (f) Ring structure formed by growing a circular Co lamella at $500{ }^{\circ} \mathrm{C}$. 


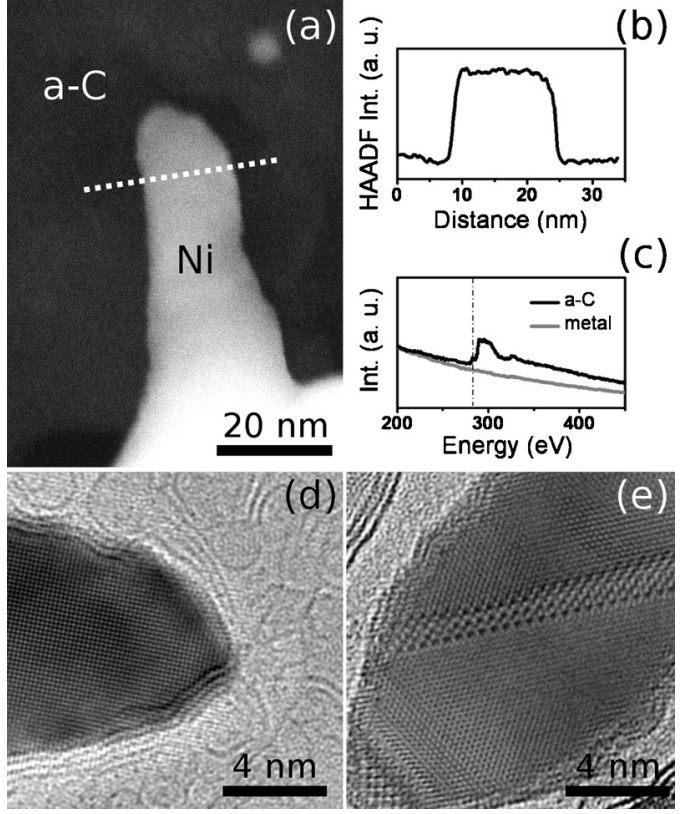

FIG. 2. (a) HAADF STEM image from a Ni lamella grown under electron irradiation from a larger Ni crystal (bottom). (b) Intensity profile of the line scan over the lamella as marked with a dotted line in (a) and showing the homogenous thickness $(3 \mathrm{~nm})$ of the metal lamella. (c) EELS spectra from an uncovered amorphous carbon film (dark line) and from a Ni lamella grown on the same film (gray line). The signal from the carbon edge (283 $\mathrm{eV}$, indicated by the dotted line) is absent in the metal region. (d) HRTEM of a Ni lamella grown at $450{ }^{\circ} \mathrm{C}$ with a [001] surface. (e) HRTEM of a Co lamella grown at $490{ }^{\circ} \mathrm{C}$ with extended twinlike defects.

directions, a pattern of metal lamellae was created as shown in Figs. 1(d) and 1(e). The growth is epitaxial with respect to the metal island, i.e., the crystal orientation of the lamella is the same as of the crystal from where growth has started. The direction of electron beam incidence relative to the bilayer system (i.e., with the metal on top or on the bottom of the carbon film) had no noticeable influence on the process.

Thicknesses of less than $5 \mathrm{~nm}$ for the thinnest metal lamellae were estimated by high-angle annular dark field (HAADF) scanning transmission electron microscopy (STEM) imaging, where a measure of the scattered intensity allows a quantitative estimate of the amount of mass under the beam. ${ }^{14}$ Figures 2(a) and 2(b) show a Ni lamella and its HAADF intensity profile, from which a homogenous thickness of $3 \mathrm{~nm}$ of the lamella can be inferred. Electron energyloss spectroscopy (EELS) showed that the amorphous carbon film had almost disappeared from the areas where the metal lamella has grown [Fig. 2(c)]. Although the structuring was induced by a $3 \mathrm{~nm}$ electron beam, the width of the metal lamellae was usually larger than $10 \mathrm{~nm}$. This is most likely due to the inherent instability of a thin metal layer with an extreme aspect ratio. High-resolution TEM images show that both $\mathrm{Co}$ and $\mathrm{Ni}$ remained crystalline during and after growth, although crystal defects occurred on some lamellae [Figs. 2(d) and 2(e)].

At temperatures above $600{ }^{\circ} \mathrm{C}$ the lamellae grew but became unstable with increasing length and, after a certain length has been exceeded, retract spontaneously toward the source crystal. At temperatures below approximately $350{ }^{\circ} \mathrm{C}$, no growth of $\mathrm{Co}$ or $\mathrm{Ni}$ was observed. In the whole temperature range up to $700{ }^{\circ} \mathrm{C}$ and under the same experi-

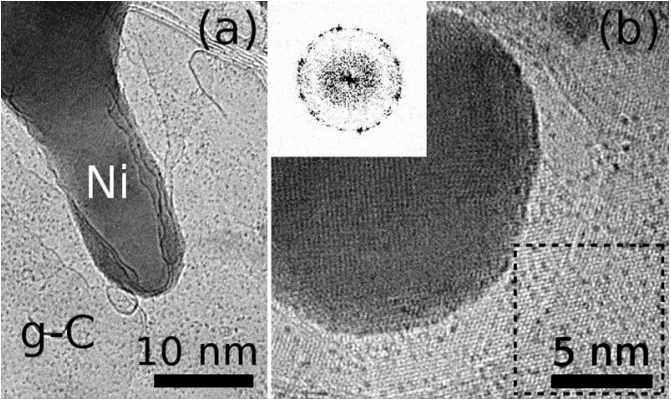

FIG. 3. (a) Ni lamella grown at $600{ }^{\circ} \mathrm{C}$ on a multilayer graphene film, labeled as $\mathrm{g}-\mathrm{C}$ in the image. (b) HRTEM image of the edge of a Ni lamella on graphene. The inset shows the Fourier transform from the dashed square region, exhibiting the distinctive hexagonal symmetry of graphenic carbon. Metals atoms (seen as small spots) appeared on the graphene surface during the irradiation process.

mental conditions, no growth of $\mathrm{Cu}$ or $\mathrm{Pt}$ layers was observable.

Co or Ni lamellae can also be grown on graphenic carbon films. This is shown in Fig. 3, where a Ni lamella has been grown from a $\mathrm{Ni}$ crystal on a multilayer graphene film. The graphene film has been made by annealing Ni crystals on an amorphous carbon film which causes the transformation of the film to graphene under suitable conditions. ${ }^{13} \mathrm{~A}$ certain minimum thickness of the carbon film is needed for the growth of metal layers. Single-layer graphene was found to be unstable against sputtering under irradiation with a 200 $\mathrm{keV}$ electron beam.

The growth of metal lamellae needs a certain mobility of the metal atoms. Since the experiment was carried out far below the melting point of the metals, ballistic atom displacements induced by energetic electrons must be the major contribution to the migration of metal atoms. Bulk Co or $\mathrm{Ni}$ atoms cannot be displaced with a $200 \mathrm{keV}$ electron beam as the displacement threshold for bulk $\mathrm{Ni}$ and $\mathrm{Co}$ is higher. ${ }^{15}$ However, metal atoms on the surface can be displaced at electron energies below the bulk threshold. ${ }^{16}$ Since displaced metal atoms migrate randomly on the surface, shape changes toward minimization of surface energy, i.e., toward a spherical shape of the metal particle would be expected. In addition, the low wetting of a perfect graphite resp. graphene surface $^{17}$ would repel metals and, again, not favor the growth of a thin lamella. However, when mobile metal atoms reach the edge of the crystals, they encounter the carbon film which has been activated by electron irradiation ${ }^{18}$ and contains enough dangling bonds that can be saturated by attaching metal atoms. Covalent metal-carbon bonds form ${ }^{19,20}$ as soon as metal atoms arrive at the interface, leading to a trapping of the metal atoms at the edge positions. Bonds between metals atoms with unfilled or partially filled d-orbitals and carbon are strong, ${ }^{20}$ e.g., with a binding energy of $7-8 \mathrm{eV}$ for bonds between carbon and $\mathrm{Co}$ or $\mathrm{Ni}^{21}$ Carbon-carbon bonds are even stronger but the supply of carbon atoms might be lower than of $\mathrm{Co}$ or $\mathrm{Ni}$ atoms. Sustained irradiation therefore leads to the growth of the metal starting from the edge. The non-appearance of the growth phenomenon for $\mathrm{Cu}$ can be explained by the low $\mathrm{C}-\mathrm{Cu}$ interaction with a bond energy of $5 \mathrm{eV}$. The $\mathrm{C}-\mathrm{Pt}$ bond has an energy of almost $8 \mathrm{eV}$ and should therefore be particularly strong. ${ }^{21}$ However, Pt atoms are much heavier than the other species of this study and therefore difficult to displace from the Pt crystals with the 
$200 \mathrm{keV}$ electron beam. In this case, the saturation of the activated carbon bonds on the surface might happen by the aggregation of migrating carbon atoms.

To conclude, the present phenomenon allows us to create predefined patterns of extremely thin metal lamellae on multilayer graphene and amorphous carbon substrates. A limitation is the inherent instability of the metal due to an increasing surface energy during growth. However, this can be overcome by keeping the temperature below the threshold for thermal relaxation of the system. Metal lamellae on multilayer graphene could be used in different applications of graphene electronics, e.g., as contacts.

Financial support by the Agence Nationale de Recherche (NANOCONTACTS, Grant No. NT09 507527) is gratefully acknowledged. The authors thank A. Krasheninnikov for helpful discussions and M. Acosta for assistance in deposition of the metal layers.

${ }^{1}$ S. J. Randolph, J. D. Fowlkes, and P. D. Rack, Crit. Rev. Solid State Mater. Sci. 31, 55 (2006).

${ }^{2}$ W. F. van Dorp and C. W. Hagen, J. Appl. Phys. 104, 081301 (2008).

${ }^{3}$ I. Utke, P. Hoffmann, and J. Melngailis, J. Vac. Sci. Technol. B 26, 1197 (2008).

${ }^{4}$ In-Situ Electron Microscopy at High Resolution, edited by F. Banhart (World Scientific, Singapore, 2008).

${ }^{5}$ M. D. Fischbein and M. Drndić, Nano Lett. 7, 1329 (2007).
${ }^{6}$ W. F. van Dorp, B. van Someren, C. W. Hagen, P. Kruit, and P. A. Crozier, Nano Lett. 5, 1303 (2005).

${ }^{7}$ S. Bysakh, M. Shimojo, K. Mitsuishi, and K. Furuya, J. Vac. Sci. Technol. B 22, 2620 (2004).

${ }^{8}$ T. Gnanavel, M. Mat Yajid, Z. Saghi, Y. Peng, B. J. Inksson, M. R. J. Gibbs, and G. Möbus, Appl. Phys. A: Mater. Sci. Process. 102, 205 (2011).

${ }^{9}$ Y. Kondo and K. Takayanagi, Phys. Rev. Lett. 79, 3455 (1997).

${ }^{10}$ H. W. Zandbergen, R. J. H. A. van Duuren, P. F. A. Alkemade, G. Lientschnig, O. Vasquez, C. Dekker, and F. D. Tichelaar, Nano Lett. 5, 549 (2005).

${ }^{11}$ S. Janbroers, T. R. de Kruijff, Q. Xu, P. J. Kooyman, and H. W. Zandbergen, Ultramicroscopy 109, 1105 (2009).

${ }^{12} \mathrm{M}$. Ohring, The Materials Science of Thin Films (Academic, London, 1991).

${ }^{13}$ J. A. Rodríguez-Manzo, C. Pham-Huu, and F. Banhart, ACS Nano 5, 1529 (2011)

${ }^{14}$ P. M. Voyles, J. L. Grazul, and D. A. Muller, Ultramicroscopy 96, 251 (2003).

${ }^{15}$ P. Jung, in Landolt-Börnstein New Series III/25: Atomic Defects in Metals, edited by H. Ullmaier (Springer, Berlin, 1991), pp. 1-87.

${ }^{16}$ R. F. Egerton, P. Li, and M. Malac, Micron 35, 399 (2004).

${ }^{17}$ M. Bilaniuk and J. M. Howe, Interface Sci. 6, 319 (1998).

${ }^{18}$ F. Banhart, Rep. Prog. Phys. 62, 1181 (1999).

${ }^{19}$ J. A. Rodríguez-Manzo, F. Banhart, M. Terrones, H. Terrones, N. Grobert, P. M. Ajayan, B. G. Sumpter, V. Meunier, M. Wang Y. Bando, and D. Golberg, Proc. Natl. Acad. Sci. U.S.A. 106, 4591 (2009).

${ }^{20}$ F. Banhart, Nanoscale 1, 201 (2009).

${ }^{21}$ A. V. Krasheninnikov, P. O. Lehtinen, A. S. Foster, P. Pyykkö, and R. M. Nieminen, Phys. Rev. Lett. 102, 126807 (2009). 